\title{
Automated quantitative MRI volumetry reports support diagnostic interpretation in dementia: a multi-rater, clinical accuracy study
}

\author{
Hugh G. Pemberton ${ }^{1,2,3}$ - Olivia Goodkin ${ }^{1,2} \cdot$ Ferran Prados $^{1,4} \cdot$ Ravi K. Das $^{5} \cdot$ Sjoerd B. Vos $^{1,2} \cdot$ James Moggridge $^{2,6}$. \\ William Coath ${ }^{3}$. Elizabeth Gordon ${ }^{3}$ - Ryan Barrett ${ }^{7}$ - Anne Schmitt ${ }^{2,6} \cdot$ Hefina Whiteley-Jones $^{7}$. Christian Burd $^{8}$. \\ Mike P. Wattjes ${ }^{9}$. Sven Haller ${ }^{10}$ • Meike W. Vernooij ${ }^{11,12}$ • Lorna Harper $^{3}$ • Nick C. Fox ${ }^{3}$ - Ross W. Paterson ${ }^{3}$. \\ Jonathan M. Schott ${ }^{3}$ - Sotirios Bisdas ${ }^{2,6}$ Mark White $^{13}$ - Sebastien Ourselin ${ }^{14}$ • John S. Thornton ${ }^{2,6}$.

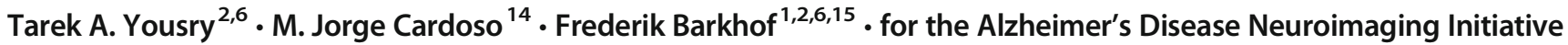

Received: 29 April 2020 / Revised: 1 October 2020 / Accepted: 2 November 2020 / Published online: 15 January 2021

(C) The Author(s) 2021

\begin{abstract}
Objectives We examined whether providing a quantitative report (QReport) of regional brain volumes improves radiologists' accuracy and confidence in detecting volume loss, and in differentiating Alzheimer's disease (AD) and frontotemporal dementia (FTD), compared with visual assessment alone.

Methods Our forced-choice multi-rater clinical accuracy study used MRI from 16 AD patients, 14 FTD patients, and 15 healthy controls; age range 52-81. Our QReport was presented to raters with regional grey matter volumes plotted as percentiles against data from a normative population $(n=461)$. Nine raters with varying radiological experience ( 3 each: consultants, registrars, 'non-clinical image analysts') assessed each case twice (with and without the QReport). Raters were blinded to clinical and demographic information; they classified scans as 'normal' or 'abnormal' and if 'abnormal' as 'AD' or 'FTD'.

Results The QReport improved sensitivity for detecting volume loss and AD across all raters combined $\left(p=0.015^{*}\right.$ and $p=0.002^{*}$, respectively). Only the consultant group's accuracy increased significantly when using the QReport ( $p=0.02 *)$. Overall, raters' agreement (Cohen's $\kappa$ ) with the 'gold standard' was not significantly affected by the QReport; only the consultant group improved significantly $\left(\kappa_{S} 0.41 \rightarrow 0.55, p=0.04 *\right)$. Cronbach's alpha for interrater agreement improved from 0.886 to 0.925 , corresponding to an improvement from 'good' to 'excellent'.

Conclusion Our QReport referencing single-subject results to normative data alongside visual assessment improved sensitivity, accuracy, and interrater agreement for detecting volume loss. The QReport was most effective in the consultants, suggesting that experience is needed to fully benefit from the additional information provided by quantitative analyses.

Key Points

- The use of quantitative report alongside routine visual MRI assessment improves sensitivity and accuracy for detecting volume loss and $A D$ vs visual assessment alone.

- Consultant neuroradiologists' assessment accuracy and agreement (kappa scores) significantly improved with the use of quantitative atrophy reports.

- First multi-rater radiological clinical evaluation of visual quantitative MRI atrophy report for use as a diagnostic aid in dementia.
\end{abstract}

Hugh Pemberton and Olivia Goodkin contributed equally.

Data used in the preparation of this article were obtained from the Alzheimer's Disease Neuroimaging Initiative (ADNI) database (adni.loni.usc.edu). As such, the investigators within the ADNI contributed to the design and implementation of ADNI and/or provided data but did not participate in the analysis or writing of this report. A complete listing of ADNI investigators can be found at: http://adni.loni.usc.edu/ wpcontent/uploads/how_to_apply/ADNI_Acknowledgement_List.pdf

Hugh G. Pemberton

h.pemberton@ucl.ac.uk

Extended author information available on the last page of the article 
Keywords Alzheimer's disease $\cdot$ Frontotemporal dementia $\cdot$ Radiologists $\cdot$ Magnetic resonance imaging $\cdot$ Atrophy

$\begin{array}{ll}\text { Abbreviations } \\ \text { AD } & \text { Alzheimer's disease } \\ \text { ADNI } & \text { Alzheimer's Disease Neuroimaging Initiative } \\ \text { BPF } & \text { Brain parenchymal fraction } \\ \text { CAU } & \text { Caudate } \\ \text { CNR } & \text { Contrast-to-noise ratio } \\ \text { FTD } & \text { Frontotemporal dementia } \\ \text { GIF } & \text { Geodesic information flows } \\ \text { GM } & \text { Grey matter } \\ \text { QNI } & \text { Quantitative neuroradiology initiative } \\ \text { QReport } & \text { Quantitative volumetry report } \\ \text { SNR } & \text { Signal-to-noise ratio } \\ \text { WM } & \text { White matter }\end{array}$

\section{Introduction}

Brain magnetic resonance imaging (MRI) is regularly used in diagnosing dementia as it visualises the structural changes caused by neurodegeneration $[1,2]$. In particular, MRI is key in defining subtle differences between healthy and pathological cerebral volume loss and between dementia subtypes [3]. These changes can be challenging to identify in both research and clinical settings, as evidenced by moderate interrater variability [4].

Several visual rating scales have been developed to enable reproducible semiquantitative assessment of volume loss [5-10]. They have been shown to reduce interrater variability to such a degree that they are used in clinical trials [11-13]. However, these scales have a subjective element and their application relies heavily on the prior experience of the radiologist using them. Furthermore, they have poor sensitivity to subtle or prodromal changes and have ceiling and/or floor effects [4]. These shortcomings can be addressed by using total and regional volume quantification, which has been used as an outcome measure in research studies and clinical trials $[11,14,15]$. It has been suggested that quantification can also improve diagnostic accuracy, reliability, confidence, and efficiency by providing regionspecific volumetric differences between single subjects and an age-matched normative population [16-21]. The clinical introduction of volume quantification is however predicated upon technical and clinical validation, as well as compliance with mandatory governance regulations [22-24].

We have developed a pipeline that automatically generates a novel and clinically usable quantitative report (QReport-Fig. 1). The segmentation algorithm we have used is Geodesic Information Flows (GIF), which is part of the in-house software NiftySeg (http://niftyweb.cs.ucl. ac.uk/program.php?p=GIF) [25]. Our pipeline integrates and displays a patient's demographic information, MRI quality control metrics, GIF's hippocampal segmentation, and volumetric results contextualised against a normative population. The QReport generates a 'rose plot' representation, which displays complex 3D data in a visually simple and easily interpreted 2D format [26]. Evaluation of most commercial reports has been limited to CE and FDA approval; this study aims to fulfil step 4 in the Quantitative Neuroradiology Initiative (QNI) six-step framework by evaluating how the QReport affects clinical accuracy [24].

In this study, we assessed the effect of our QReport across two diagnostic steps and three neuroradiological levels of experience. We hypothesised (1) that the use of our QReport will decrease interrater variability whilst increasing diagnostic specificity, sensitivity, accuracy, and confidence (a) for determining the presence of volume loss and (b) for determining the differential diagnosis of AD or FTD; and (2) that the QReport's effect will be identifiable across the three experience levels.

\section{Methods}

\section{Patient dataset}

We established a test set of MRI scans from 45 subjects scanned locally, using three different 3-T MRI systems (see supplementary material for acquisition parameters). Fifteen 'control subjects' were referred to our specialist clinic on memory concerns but deemed to fall within normal ranges upon neurological, cerebrospinal fluid (CSF) and imaging assessment. MMSE scores have been included as a marker of cognitive performance (see Table 1).

Fig. 1 Quantitative report (QReport) of an AD patient displaying demographics, hippocampal volume percentiles, and single-subject brain parenchymal fraction (red dot) plotted against a normative dementia-free population. Quality control metrics and a 'rose plot' representation of GM volume percentiles split by brain lobe and relevant sub-regions. The rose plot is on a log scale and uses a traffic light colour-coding system (green to red meaning high to low percentile) to display the individual's volume percentiles in the context of a healthy population. Abbreviations: BPF, brain parenchymal fraction; SNR, signal-to-noise ratio; CNR, contrast-to-noise ratio; GM, grey matter; $\mathrm{WM}$, white matter; CAU, caudate 

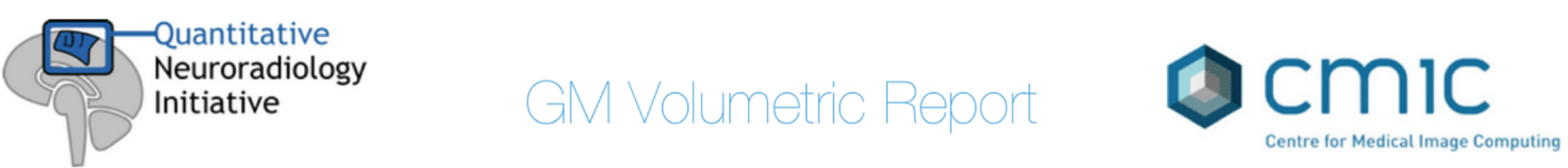

\section{PATIENT INFORMATION \& GLOBAL ANALYSIS}

\begin{tabular}{l|c|c|c|c|c} 
Name & Hospital ID & Age / Gender & Scan Date & Scanner & Hospital \\
NiftyWeb & NiftyWeb & $56.3 /$ Female & 3 Dec 2015 & Siemens (3T) & Somewhere
\end{tabular}

HIPPOCAMPAL VOLUME PERCENTILE Percentile (pctl) compared to a healthy population

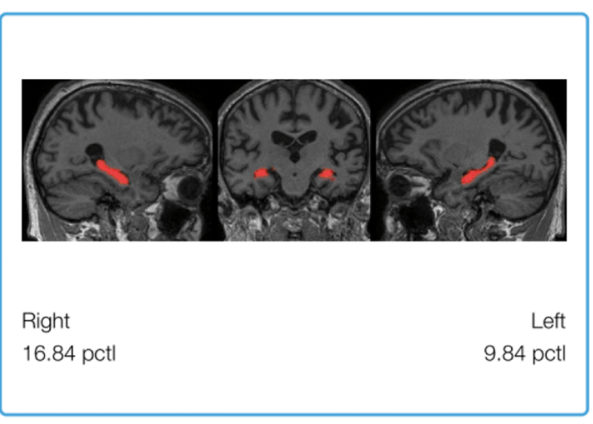

BRAIN PARENCHYMAL FRACTION

Brain volume as percentage of total intracranial volume

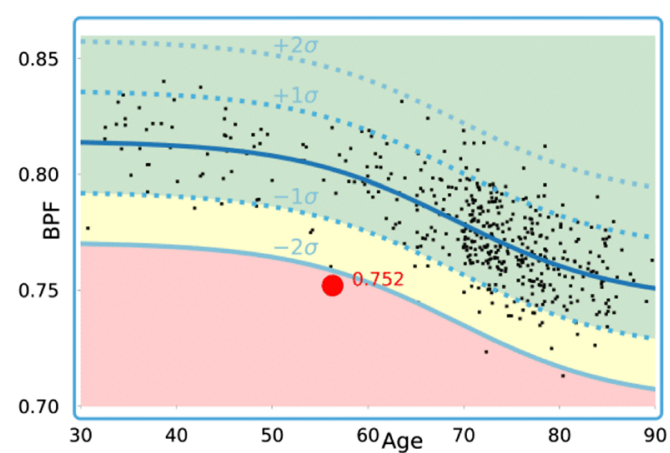

QUALITY CONTROL Automated Scores

\begin{tabular}{|c|c|c|}
\hline Metric & Type & Status \\
\hline SNR & WM & Pass \\
\hline CNR & GMWM & Pass \\
\hline Artefact & $\begin{array}{c}\text { Movement } \\
\text { Aliasing }\end{array}$ & \\
\hline Algorithm & Model Fit & Pass \\
\hline
\end{tabular}

REGIONAL ANALYSIS

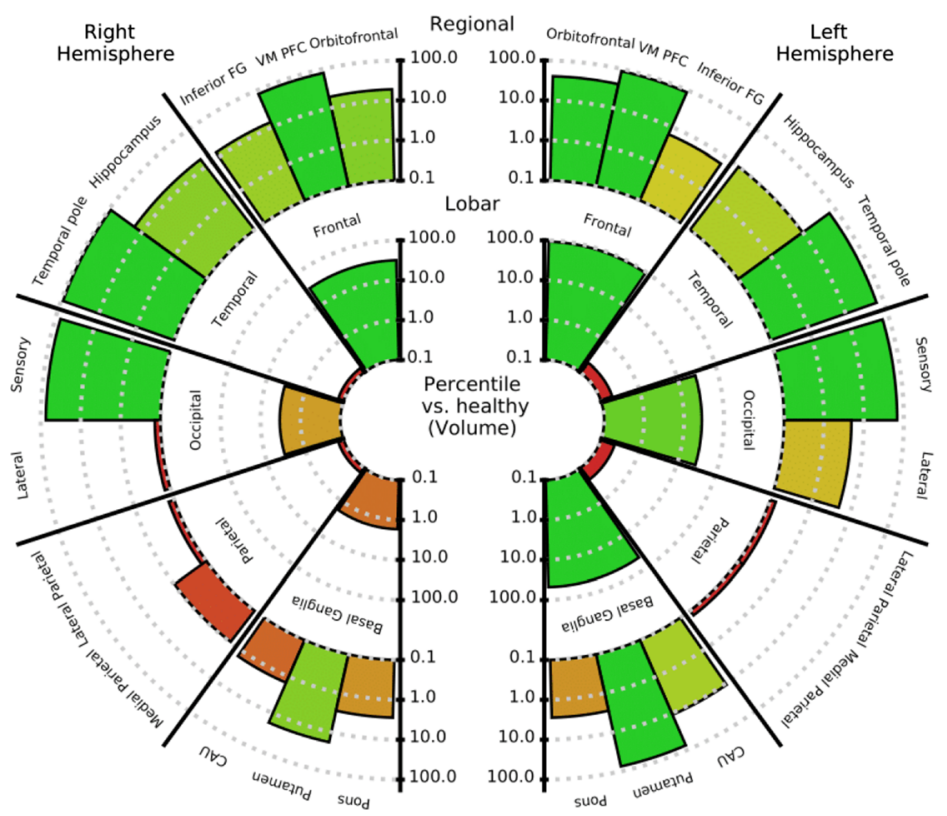

Thirty patients were diagnosed with either AD $(n=16$, beta-amyloid $1-42<550 \mathrm{pg} / \mathrm{mL}$ and tau:amyloid ratio $>1$ ) or FTD $(n=14)$, based on clinical evaluation and CSF markers. MMSE scores and disease duration are provided in 
Table 1 Characteristics of the test subject data set. Mean age was matched across subjects, mean Abeta 1-42 was reduced and mean Tau was raised for AD subjects relative to controls. Mean MMSE was significantly lower for AD $(p<0.001)$ and FTD $(p=0.03)$ when compared with 'controls'. Mean disease duration (time from first reported symptom to MRI) in y is also shown

\begin{tabular}{lcccc}
\hline & Controls $(n=15)$ & AD $(n=16)$ & FTD $(n=14)$ & Total $(n=45)$ \\
\hline Age in years, mean (SD) & $60(8.7)$ & $61.7(6.6)$ & $59.9(7.3)$ & $60.6(7.4)$ \\
Gender male:female & $4: 11$ & $9: 7$ & $11: 3$ & $24: 21$ \\
Mean Abeta 1-42 (pg/mL) & 878.8 & 393.3 & 747.7 & - \\
Mean Tau (pg/mL) & 373.3 & 855.2 & 302.6 & - \\
MMSE, mean (SD) & $26.9(4)$ & $20.5(6.4)$ & $22(9.1)$ & - \\
Disease duration in years, mean (SD) & - & $2.7(1.6)$ & $3.5(2.4)$ & - \\
\hline
\end{tabular}

Table 1. All data were acquired under ethical approval by the Queen Square ethics committee: 13 LO 0005 and 12 LO 1504.

\section{Reference dataset}

The normative healthy control data were derived from the Alzheimer's Disease Neuroimaging Initiative (ADNI) database ( $n=382$; age range 56-90 years) (adni.loni.usc.edu) augmented by the Track-HD study cohort [27] to include younger controls ( $n=79$; age range $=30-65$ years $)$ thereby covering a clinically appropriate age range. The total normative population was $n=461$ ( $51.4 \%$ female), and the mean age was 70.09 years, $\mathrm{SD}=12.05$. Subject data in the 'reference dataset' were acquired under the ethical agreements in place for ADNI and TRACK-HD studies.

\section{Quantification and display of grey matter volumes}

Whole brain, grey matter, and relevant regional volumes were estimated for all participants using Geodesic Information Flows (GIF). GIF provides fully automated multi-atlas segmentation and global and region-specific volumetry of T1-weighted scans. It has been validated in manual segmentation studies both in dementia and other neurodegenerative disorders [25, 28-30]. This is especially relevant for the comparison of morphologically different subjects, as examined in this study [25, 31]. We developed an automated pipeline that presents data in a clinically usable report format (Fig. 1) displaying non-identifying demographics, hippocampal volume percentiles, and brain parenchymal fraction plotted against normative population data. Regional brain volumes were expressed as percentile estimates against a Gaussian

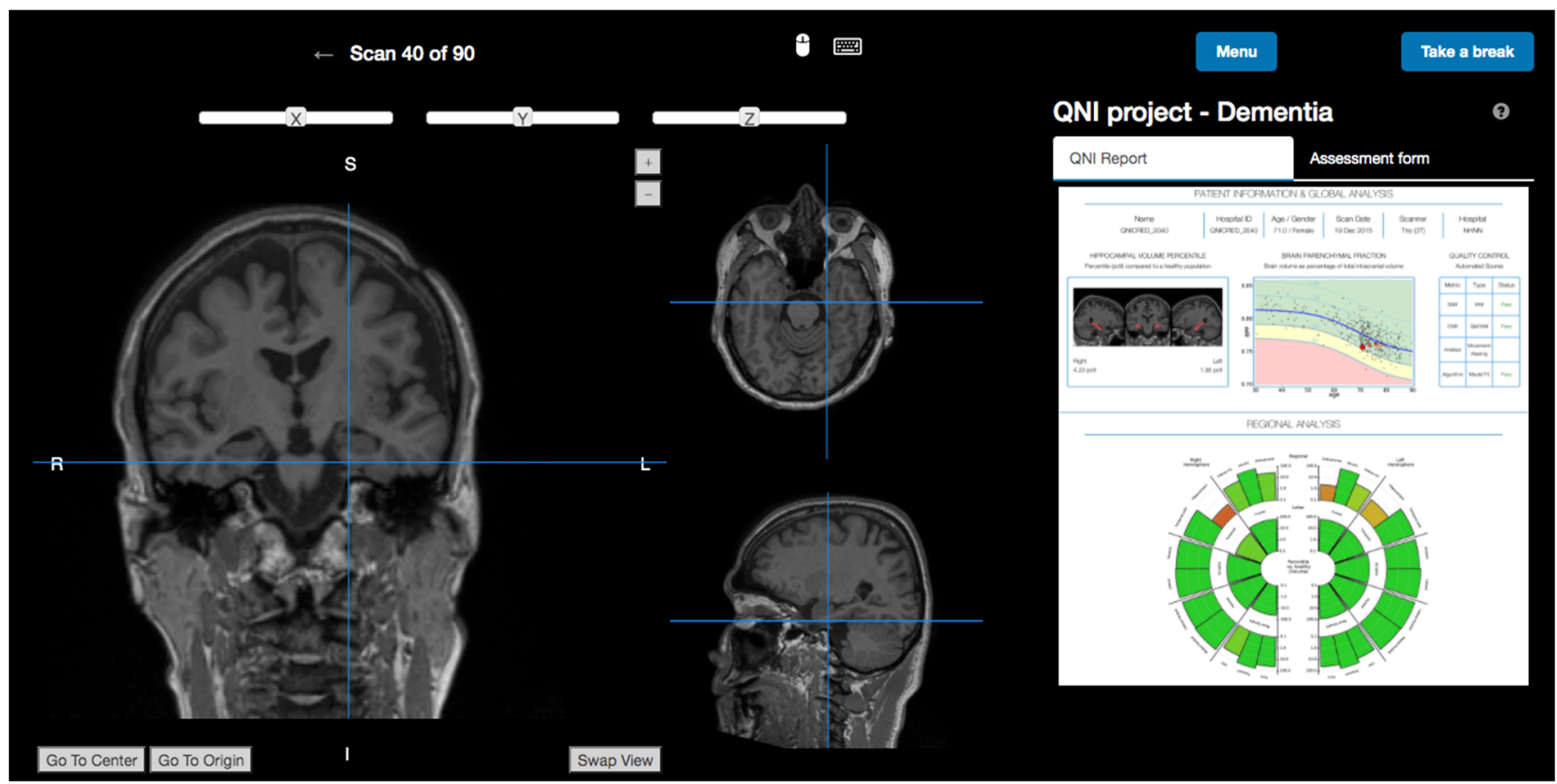

Fig. 2 Screenshot from the Quantitative Neuroradiology Initiative (QNI) study website (http://qni.cs.ucl.ac.uk) showing the image viewer for a case with the QReport available. QReports were fully interactive and zoomable via the website 
distribution approximation of healthy control grey matter volumes, after regressing out age, gender, and total intracranial volume. We used a variant of a generalised logistic function to predict the values of our observational normative database as a continuous variable. This allowed us to compute the cumulative distribution function of measured values with respect to the normative population. Data were displayed in a visually simple and intuitive 'rose plot' format.

\section{Study design}

Three groups of raters participated in this study: consultant neuroradiologists; neuroradiology specialty registrars; and non-clinical image analysts. Raters were invited from multiple centres, ensuring a broad representation of training and experience. Raters were blinded to all clinical and demographic information except for age and gender. We designed a website platform (Fig. 2) to facilitate remote participation. The website included thorough instructions (see supplementary material) followed by 45 scans displayed once with and once without the QReport available. In order to mitigate against systematic learning or anchoring effects, scans were automatically randomised and delivered to raters in a unique order per rater through our rating website. The task consisted of 90 evaluation 'episodes' in total.

At each 'episode', raters were prompted to give their assessment, stating (1.a) whether the scan was 'normal' or 'abnormal' in terms of volume loss for age; (1.b) degree of confidence on a scale of 1 (very uncertain) to 5 (very confident); (2.a) if the scan was rated abnormal, to select AD or FTD; and (2.b) their confidence level for this differential diagnosis (1-5 scale). Raters completed the exercise over a period of 2 months; ratings were collected through the web platform and subsequently analysed.

\section{Statistical analysis}

We explored the effects of QReport availability on the accuracy of (1) identifying volume loss (normal versus abnormal) and (2) differential diagnosis of $\mathrm{AD}$ versus FTD. Key signal-detection indices were calculated using the following ratings: (a) correctly defined as 'abnormal' ('true positive' for $\mathrm{AD} / \mathrm{FTD}$ ), and 'normal' ('true negative' for healthy controls) and (b) incorrectly defined as 'abnormal' ('false positive' for healthy controls) and 'normal' ('false negative' for patients). Using these metrics, diagnostic sensitivity, specificity, and accuracy were calculated and expressed as percentages as follows:
Accuracy
$=\frac{\text { True Positives }+ \text { True Negatives }}{\text { True Positives }+ \text { True Negatives }+ \text { False Positives }+ \text { False Negatives }} \times 100$

Sensitivity $=\frac{\text { True Positives }}{\text { True Positives }+ \text { False Negatives }} \times 100$

Specificity $=\frac{\text { True Negatives }}{\text { True Negatives }+ \text { False Positives }} \times 100$

Subsequently, counts of correctly and incorrectly diagnosed scans with and without the QReport available were analysed with the McNemar test. Paired $t$ tests were used to assess mean diagnostic accuracy, specificity, and sensitivity across the two conditions (QReport present vs absent). Cohen's kappa was calculated to assess agreement between raters' evaluations and confirmed diagnosis while accounting for 'chance' agreement. To further assess the effect of the QReport's availability on consistency and reliability among raters, Cronbach's alpha and intraclass correlation coefficients were calculated.

Confidence ratings (QReport vs no QReport) were calculated as a grand mean per rater and for each 'true' disease type (normal, AD, FTD) and assessed with paired $t$ tests. In exploratory analyses rating, normal vs abnormal, we hypothesised that the effects of the QReport on confidence and diagnostic ratings could vary depending on whether the rated scans were normal or abnormal and whether the raters correctly classified the scans and the experience level of the raters. A fourway mixed ANOVA, including all factors (QReport $\times$ normality $\times$ correctness $\times$ experience level), allowed us to assess how these factors interact.

All statistical analyses were performed with SPSS version 24 .

\section{Results}

\section{Assessment accuracy}

\section{Volume loss: normal vs abnormal}

For all raters combined, the availability of the QReport significantly improved the diagnostic sensitivity $(p=$ $\left.0.015^{*}\right)$, without changing the specificity or accuracy. However, for accuracy, a beneficial medium effect size (0.53) was observed. Of the 3 rating groups, only the consultant group's accuracy improved significantly, from 71 to $80 \%(p=0.02 *$ (Table 2$)$. 
Table 2 Sensitivity, specificity, and accuracy for normal vs abnormal rating across all experience levels, both with and without the quantitative report

\begin{tabular}{|c|c|c|c|c|c|}
\hline Metric & Experience level & Without report mean (SD) & With report mean (SD) & $p$ value & $d$ effect size \\
\hline \multirow[t]{4}{*}{ Sensitivity } & Consultant & $68.9 \%(5)$ & $80 \%(10)$ & 0.13 & 1.4 \\
\hline & Registrar & $75.5 \%(8.4)$ & $81.1 \%(1.9)$ & 0.3 & 0.8 \\
\hline & Image analyst & $70 \%(25.1)$ & $85.5 \%(10.1)$ & 0.23 & 0.9 \\
\hline & All groups combined & $71.5 \%(13.8)$ & $82.2 \%(7.6)$ & $0.015^{*}$ & 1.03 \\
\hline \multirow[t]{4}{*}{ Specificity } & Consultant & $75.6 \%(3.8)$ & $80 \%(13.3)$ & 0.52 & 0.43 \\
\hline & Registrar & $82.2 \%(10.1)$ & $68.8 \%(25.2)$ & 0.37 & -0.6 \\
\hline & Image analyst & $77.7 \%(3.8)$ & $68.9 \%(13.8)$ & 0.45 & -0.52 \\
\hline & All groups combined & $78.5 \%(6.4)$ & $72.3 \%(16.8)$ & 0.3 & -0.37 \\
\hline \multirow[t]{4}{*}{ Accuracy } & Consultant & $71.1 \%(2.2)$ & $80 \%(2.2)$ & $0.02 *$ & 4 \\
\hline & Registrar & $77.7 \%(3.8)$ & $77 \%(9.2)$ & 0.87 & -0.1 \\
\hline & Image analyst & $72.6 \%(17.9)$ & $80 \%(2.2)$ & 0.5 & 0.46 \\
\hline & All groups combined & $73.8 \%(9.5)$ & $79 \%(5.1)$ & 0.15 & 0.53 \\
\hline
\end{tabular}

* Statistically significant at $<0.05$

\section{$A D$ vs FTD}

The presence of the QReport significantly improved sensitivity for $\mathrm{AD}$ in the image analysts $(p=0.01 *)$ and for all raters combined $(p=0.002 *)$ (Table 3$)$. There were no significant changes in diagnosing FTD (Table 4). In absolute terms, the number of correct diagnoses of AD and FTD increased with the report by $6.9 \%$ and $5.6 \%$, respectively, with a medium effect size for $\mathrm{AD}$, but these changes were not significant.

\section{Assessment confidence}

For rating normal vs abnormal, using a four-way mixed ANOVA (QReport $\times$ normality $\times$ correctness $\times$ experience level), we found a normality $\times$ correctness $\times$ QReport interaction, indicating significantly increased confidence when incorrectly rating abnormal scans with the QReport (i.e. false-positive judgement). These findings represent a statistically significant difference $(p=0.02$ and $F(1,8)=7.918)$, with a small effect size $\left(\eta_{\mathrm{p}}^{2}=0.497\right)$, which did not vary across experience level groups. Raters were also significantly more confident:

1. With the QReport than without, regardless of correctness $\left[F(1,8)=6.64, p=0.03, \eta_{\mathrm{p}}^{2}=0.453\right]$

2. When correctly rating, regardless of QReport use $\left[F(1,8)=112.43, p=<0.01, \eta_{\mathrm{p}}^{2}=0.934\right]$

3. When rating abnormal rather than normal scans, regardless of QReport use $\left[F(1,8)=21.68, p=<0.01, \eta_{\mathrm{p}}^{2}=\right.$ $0.73]$

Table 3 Sensitivity, specificity, and accuracy for AD vs normal rating across all experience levels, and percentage of correct assessments for AD, both with and without the quantitative report

\begin{tabular}{|c|c|c|c|c|c|}
\hline Metric & experience level & Without report mean (SD) & With report mean (SD) & $p$ value & $d$ effect size \\
\hline \multirow[t]{4}{*}{ Sensitivity } & Consultant & $61.3 \%(12.9)$ & $75.8 \%(17)$ & 0.05 & 0.96 \\
\hline & Registrar & $79.3 \%(12.7)$ & $83.9 \%(4.7)$ & 0.42 & 0.48 \\
\hline & Image analyst & $61.7 \%(45.1)$ & $71.1 \%(44.4)$ & $0.01 *$ & 0.22 \\
\hline & All groups combined & $67.4 \%(25.8)$ & $76.9 \%(24.5)$ & $0.002 *$ & 0.37 \\
\hline \multirow[t]{4}{*}{ Specificity } & Consultant & $79.6 \%(8)$ & $82 \%(14.6)$ & 0.73 & 0.2 \\
\hline & Registrar & $83.1 \%(7.5)$ & $78.1 \%(7.8)$ & 0.46 & -0.65 \\
\hline & Image analyst & $86.6 \%(4.2)$ & $77.9 \%(12.9)$ & 0.31 & -0.9 \\
\hline & All groups combined & $83.1 \%(6.6)$ & $79.3 \%(10.7)$ & 0.3 & 0.42 \\
\hline \multirow[t]{4}{*}{ Accuracy } & Consultant & $70.7 \%(3.2)$ & $79.2 \%(10.9)$ & 0.07 & 1.05 \\
\hline & Registrar & $80.3 \%(2.3)$ & $78.9 \%(7.4)$ & 0.76 & -0.25 \\
\hline & Image analyst & $75.8 \%(16.8)$ & $77.9 \%(4.2)$ & 0.84 & 0.17 \\
\hline & All groups combined & $75.5 \%(9.6)$ & $78.7 \%(4.3)$ & 0.38 & 0.43 \\
\hline \multicolumn{2}{|c|}{ Correct AD diagnoses } & $58.1 \%(3.4)$ & $65 \%(4.1)$ & 0.128 & 0.56 \\
\hline
\end{tabular}

* Statistically significant at $<0.05$ 
Table 4 Sensitivity, specificity, and accuracy for FTD vs normal rating across all experience levels, and percentage of correct assessments for FTD, both with and without the quantitative report

\begin{tabular}{|c|c|c|c|c|c|}
\hline Metric & Experience level & Without report mean (SD) & With report mean (SD) & $p$ value & $d$ effect size \\
\hline \multirow[t]{4}{*}{ Sensitivity } & Consultant & $57.3 \%(4.1)$ & $57.2 \%(6.2)$ & 0.93 & -0.01 \\
\hline & Registrar & $36.5 \%(7.8)$ & $35.2 \%(23.8)$ & 0.94 & -0.07 \\
\hline & Image analyst & $46.9 \%(24.9)$ & $58.3 \%(20.2)$ & 0.1 & 0.5 \\
\hline & All groups combined & $46.9 \%(16)$ & $50.3 \%(19.5)$ & 0.52 & 0.19 \\
\hline \multirow[t]{4}{*}{ Specificity } & Consultant & $89.2 \%(9.4)$ & $95 \%(6.5)$ & 0.19 & 0.71 \\
\hline & Registrar & $91.1 \%(9.7)$ & $77.7 \%(32.7)$ & 0.42 & -0.55 \\
\hline & Image analyst & $75.5 \%(28.9)$ & $85.5 \%(14.5)$ & 0.46 & 0.43 \\
\hline & All groups combined & $85.2 \%(17.6)$ & $86.1 \%(19.7)$ & 0.89 & 0.04 \\
\hline \multirow[t]{4}{*}{ Accuracy } & Consultant & $73.6 \%(4.9)$ & $75.9 \%(3.6)$ & 0.09 & 0.53 \\
\hline & Registrar & $70.5 \%(11)$ & $65.2 \%(22.8)$ & 0.52 & 0.29 \\
\hline & Image analyst & $69.1 \%(15.9)$ & $72.6 \%(14.3)$ & 0.41 & 0.23 \\
\hline & All groups combined & $71.1 \%(10.2)$ & $71.2 \%(14.4)$ & 0.95 & 0.01 \\
\hline \multicolumn{2}{|c|}{ Correct FTD diagnoses } & $38.6 \%(2.2)$ & $44.2 \%(2.7)$ & 0.367 & 0.31 \\
\hline
\end{tabular}

There were no other significant effects on confidence when using the QReport.

\section{Agreement between raters and gold standard-Kappa scores}

Cohen's kappa scores for each rater when detecting volume loss (abnormal) are detailed in Table 5, and for differentiating between AD or FTD in Table 6. For both assessments, only the consultant group's kappa scores increased significantly when using the QReport ( $p=0.038^{*}$ and $p=0.04^{*}$, respectively).

\section{Agreement and reliability across raters}

For rating normal vs abnormal, Cronbach's alpha for agreement across all raters showed improvement in

Table 5 Kappa scores for normal/abnormal assessments across all experience levels, both with and without the quantitative report

\begin{tabular}{llllll}
\hline Experience level & Rater\# No report & $\begin{array}{l}\text { With } \\
\text { report }\end{array}$ & $\begin{array}{l}\text { Net } \\
\text { change }\end{array}$ & $\begin{array}{l}p \\
\text { value }\end{array}$ \\
\hline Consultant & $\mathrm{A} 1$ & 0.400 & 0.586 & 0.186 & $0.038^{*}$ \\
& $\mathrm{~A} 2$ & 0.469 & 0.571 & 0.102 & \\
& $\mathrm{~A} 3$ & 0.381 & 0.492 & 0.111 & \\
Registrar & $\mathrm{B} 1$ & 0.455 & 0.211 & -0.244 & 0.68 \\
& $\mathrm{~B} 2$ & 0.522 & 0.571 & 0.05 & \\
\multirow{2}{*}{ Image analyst } & $\mathrm{B} 3$ & 0.613 & 0.667 & 0.054 & \\
& $\mathrm{C} 1$ & 0.169 & 0.531 & 0.362 & 0.66 \\
& $\mathrm{C} 2$ & 0.746 & 0.556 & -0.19 & \\
\multirow{2}{*}{ Overall Mean (SD) } & $\mathrm{C} 3$ & 0.492 & 0.557 & 0.065 & \\
\hline
\end{tabular}

*Statistically significant at $<0.05$ overall rating reliability from 0.886 to 0.925 with the QReport available, corresponding to an improvement from 'good' to 'excellent'. The intraclass correlation coefficient, assessed using mixed two-way ANOVA across raters, was 0.454 for single measures and 0.882 for average measures; with the QReport, these increased to 0.563 and 0.921 , respectively.

\section{Power calculations}

Based on our observed effect sizes of diagnostic accuracy (Table 2) for all raters, we have calculated the following sample size estimations to help inform future studies. To achieve an $80 \%, 90 \%$, and $95 \%$ chance of observing a positive effect, 30,40 , and 45 raters would be required, respectively.

Table 6 Kappa scores for agreement between rated diagnosis and clinically/CSF-confirmed AD and FTD diagnoses across all experience levels, both with and without the quantitative report

\begin{tabular}{|c|c|c|c|c|c|}
\hline Experience Level & Rater\# & No report & $\begin{array}{l}\text { With } \\
\text { report }\end{array}$ & $\begin{array}{l}\text { Net } \\
\text { change }\end{array}$ & $\begin{array}{l}p \\
\text { value }\end{array}$ \\
\hline \multirow[t]{3}{*}{ Consultant } & $\mathrm{Al}$ & 0.432 & 0.531 & 0.099 & \multirow[t]{3}{*}{$0.04 *$} \\
\hline & $\mathrm{A} 2$ & 0.45 & 0.498 & 0.048 & \\
\hline & A3 & 0.335 & 0.434 & 0.099 & \\
\hline \multirow[t]{3}{*}{ Registrar } & B1 & 0.381 & 0.22 & -0.161 & \multirow[t]{3}{*}{0.56} \\
\hline & B2 & 0.326 & 0.428 & 0.102 & \\
\hline & B3 & 0.494 & 0.391 & -0.103 & \\
\hline \multirow[t]{3}{*}{ Image analyst } & $\mathrm{Cl}$ & 0.02 & 0.176 & 0.156 & \multirow[t]{3}{*}{0.28} \\
\hline & $\mathrm{C} 2$ & 0.529 & 0.496 & -0.033 & \\
\hline & $\mathrm{C} 3$ & 0.396 & 0.529 & 0.133 & \\
\hline Overall Mean (SD) & & $0.37(0.15)$ & $0.41(0.13)$ & 0.037 & 0.39 \\
\hline
\end{tabular}

*Statistically significant at $<0.05$ 


\section{Discussion}

We performed a clinical accuracy study of our quantitative volumetric report (QReport-Fig. 1). Using an established segmentation algorithm, Geodesic Information Flows (GIF) [25], we developed a pipeline that brings together patient demographic information, hippocampal segmentation, brain parenchymal fraction, and global- and regionspecific brain volumetry contextualised against a normative population (Fig. 1). The advantage of our 'rose plot' display is the representation of complex 3D data in a visually simple and easily interpretable 2D format. Our main aim was to assess the effect of our novel quantitative volumetric report on sensitivity, specificity, and accuracy across three neuroradiological levels of experience. Providing our QReport increased the sensitivity of detecting volume loss across all raters and improved the accuracy and agreement among the consultant group. It also improved sensitivity for diagnosing $\mathrm{AD}$ in the image analysts and for all raters combined, but had no effect on FTD discrimination. Further to this, the QReport reduced the variability in accuracy, sensitivity, and kappa scores for detecting volume loss. In absolute terms, the classification accuracy increased overall by over $5 \%$. Given the documented increases in dementia prevalence in recent years and its future projections [32], this figure could be of clinical importance if confirmed in a larger study population.

Proprietary quantitative tools exist for the assessment of dementia, such as CorTechs.AI's 'Neuroquant' (https:// www.cortechs.ai/products/neuroquant/tbi/) and Icometrix's 'icobrain-dm' (https://icometrix.com/products/icobrain-dm). Technical validation of their segmentation algorithms has been performed versus other segmentation procedures, with promising results [33, 34]. However, systematic assessment of their clinical accuracy by neuroradiologists, as addressed in the current study, has not been published for either, despite both tools being FDA and CE approved. Our 'rose plot' provides more intuitive information than numerical tables of sub-region volumes and limited visualisations of lobar and hippocampal volumes alone. There is a major lack of clinical validation studies in the literature for volumetric neuroradiological tools. In line with our research, a recent study showed improved identification of patients versus healthy controls for one of two raters, while both raters improved in the differential diagnosis of 'dementing neurodegenerative disorders' [21].

In a study using non-commercial algorithms, it was shown that adding lobar and hippocampal volumes to visual inspection improved the diagnostic accuracy of two experienced neuroradiologists [19], thereby mirroring our findings. This improvement suggests that experienced neuroradiologists are well placed to assimilate and make use of the information provided by the QReport. Furthermore, our consultant group showed the greatest statistical benefit due to having the least variance in their assessment performance between the two tasks, which is to be expected especially when compared with the non-clinical group (Table 2). Conversely, it is possible that less experienced neuroradiologists and non-clinical image analysts were over-reliant on the QReport for determining abnormality, as suggested by an overall decrease in specificity, although not significant (Tables 2 and 3).

When diagnosing a neurodegenerative disease on MR images, neuroradiologists first assess the presence of volume loss as well as its distribution. In a second step, they interpret the pattern to be indicative of a certain disease type, such as $\mathrm{AD}$ or FTD. In this context, it is worth noting that providing the QReport increased the sensitivity of the first step (the detection of volume loss across all raters) and improved the accuracy and agreement among the consultant group. For the differential diagnosis, the QReport improved sensitivity for $\mathrm{AD}$ in the image analysts and for all raters combined but had no effect on FTD. From a diagnostic point of view, providing an objective measure to reproducibly assess volume loss with a decreased interrater variability is crucial and could be used clinically in a number of neurodegenerative diseases. The limited effects on the differential diagnosis on FTD could be due to the low mean age of patients (61.7 years for $\mathrm{AD}$ and 59.9 years for FTD) and relatively short disease durations ( 2.7 years for $\mathrm{AD}$ and 3.5 years for FTD) (Table 1). This will have affected the degree of atrophy present and possibly made them harder cases to assess. However, it is also important to identify atrophy in younger patients while it is still subtle, and it is in these cases especially where a QReport could help reduce subjective visual disagreement.

Interestingly, confidence in detecting volume loss and differentiating AD and FTD was not significantly affected by the QReport. Significantly increased confidence was unexpectedly shown when incorrectly diagnosing volume loss (i.e. false confidence) independent of experience level. One potential explanation is that raters based their incorrect diagnosis on visual inspection alone and used the QReport to reinforce their diagnosis. Irrespective of the reason, more work needs to be done to understand and mitigate this finding. It highlights the need for rigorous validation before clinical adoption and the importance of appropriate training to avoid over-reliance on diagnostic aids, completion of a test case set, and carefully planning and monitoring the introduction of tools such as the QReport. Rather than a gold standard, quantitative reports should be considered support tools which cannot replace neuroradiological experience, and raters should be wary of over-reliance.

\section{Limitations}

Our study was somewhat limited in statistical power, due potentially to the subject sample size or the number of raters used. However, our sample size of 45 subjects was in line with other similar studies using between 36 and 52 subjects [17, 19, 20]. The use of nine raters within three experience levels enabled us to 
identify the effect of experience when introducing QReports. Similar work has used a total of 2 raters $[19,20]$ or a maximum of 3 raters [17]. The performance of our image analyst group was unexpectedly heterogeneous, likely due to disparity in experience level. The variability in the results within the image analysts and registrar groups could also reflect an over-reliance on the report, rather than using it in addition to the MRI. The 'control' group was half the size of the patient group, which could have contributed to unexpected decreases in specificity, although not significant (Tables 2 and 3). Our study therefore underlines the importance of considering sample sizes and rater groups when developing and validating such quantitative diagnostic aids. Future work will need to recruit more raters to better assess the effects of the report in diagnostic performance, and the moderators of this effect (see "Power calculations" in the "Results" section).

The 'control' subjects were recruited from a clinical population who all presented with subjective neurological complaints. It is possible that radiologically normal 'controls' had other pathologies, which may have affected our raters' performance. This was, however, a conscious choice to reflect the clinical setting in memory clinics. Finally, the incidence ratio (Controls:AD:FTD), forced-choice nature, and lack of further clinical data in this study are not a reflection of routine neuroradiological assessment where more diagnostic options need to be considered.

\section{Conclusions}

The results of this clinical accuracy study demonstrate that quantitative volume reports providing single-subject results referenced to normative data can improve the sensitivity, accuracy, and inter-observer agreement for detecting volume loss and AD. This is a crucial step when reporting volume changes in patients with dementia. The largest beneficial effect of the QReport was in the consultant group, suggesting they were best placed to assimilate and make use of the information provided by the QReport. The differing effects between all three experience levels highlight the need for studies clarifying the potential benefits and limitations of these reports, and the importance of rigorous validation before clinical adoption. Our sample sizes were low, but the effect sizes across accuracy and sensitivity were moderate-to-large in favour of a beneficial report effect. Importantly, a reduced variability in sensitivity, accuracy, and kappa scores was also noted. We believe our study will help to inform power calculations and study design for future research in the field.

\section{Software availability}

The software is non-commercial and a QReport can be freely generated by uploading a T1-weighted scan via this weblink—http://niftyweb.cs.ucl.ac.uk/program.php?p= QNID.
Supplementary Information The online version contains supplementary material available at https://doi.org/10.1007/s00330-020-07455-8.

Acknowledgements The authors would like to thank all the study participants. We would also like to thank Rachel Scahill and Sarah Tabrizi for use of their TRACK-HD data. Data used in the preparation of this article were obtained in part from the Alzheimer's Disease Neuroimaging Initiative (ADNI) database (adni.loni.usc.edu). Data collection and sharing for this project were funded by the Alzheimer's Disease Neuroimaging Initiative (ADNI) (National Institutes of Health Grant U01 AG024904) and DOD ADNI (Department of Defense award number W81XWH-12-2-0012). The ADNI was launched in 2003 as a publicprivate partnership, led by Principal Investigator Michael W. Weiner, MD. The primary goal of ADNI has been to test whether serial magnetic resonance imaging (MRI), positron emission tomography (PET), other biological markers, and clinical and neuropsychological assessment can be combined to measure the progression of mild cognitive impairment (MCI) and early Alzheimer's disease (AD). ADNI is funded by the National Institute on Aging, the National Institute of Biomedical Imaging and Bioengineering, and through generous contributions from the following: AbbVie, Alzheimer's Association; Alzheimer's Drug Discovery Foundation; Araclon Biotech; BioClinica, Inc.; Biogen; Bristol-Myers Squibb Company; CereSpir, Inc.; Cogstate; Eisai Inc.; Elan Pharmaceuticals, Inc.; Eli Lilly and Company; EuroImmun; F. Hoffmann-La Roche Ltd. and its affiliated company Genentech, Inc.; Fujirebio; GE Healthcare; IXICO Ltd.; Janssen Alzheimer Immunotherapy Research \& Development, LLC.; Johnson \& Johnson Pharmaceutical Research \& Development LLC.; Lumosity; Lundbeck; Merck \& Co., Inc.; Meso Scale Diagnostics, LLC.; NeuroRx Research; Neurotrack Technologies; Novartis Pharmaceuticals Corporation; Pfizer Inc.; Piramal Imaging; Servier; Takeda Pharmaceutical Company; and Transition Therapeutics. The Canadian Institutes of Health Research is providing funds to support ADNI clinical sites in Canada. Private sector contributions are facilitated by the Foundation for the National Institutes of Health (www.fnih.org). The grantee organisation is the Northern California Institute for Research and Education, and the study is coordinated by the Alzheimer's Therapeutic Research Institute at the University of Southern California. ADNI data are disseminated by the Laboratory for Neuro Imaging at the University of Southern California.

Funding HP is funded by the Engineering and Physical Science Research Council (EPSRC). FB, SBV, OG, TAY, and JT are funded by the NIHR Biomedical Research Centre at University College London Hospital. FP has a non-clinical Guarantors of Brain fellowship. SO and MJC are funded by the EPSRC, the Wellcome Trust, the Wolfson Foundation, and the NIHR Biomedical Research Centre at Guy's and St Thomas' Trust. Further funding for the project came from the Leonard Wolfson Experimental Neurology Centre at Queen Square. The Dementia Research Centre is supported by Alzheimer's Research UK, Brain Research Trust, and The Wolfson Foundation. RWP is supported by an Alzheimer's Association Clinician Scientist fellowship, the UK Dementia Research Institute, and the UCL Queen Square Biomedical Research Centre.

\section{Compliance with ethical standards}

Guarantor The scientific guarantor of this publication is Prof Frederik Barkhof.

Conflict of interest SH is a speaker for General Electric, a consultant for Spineart and on the imaging advisory board for the European Prevention of Alzheimer's Disease. JC and SO are founders of Brainminer. MPW received speaker and/or consultancy fees from Bayer, Biogen, Biologix, Celgene, GeniLac, Imcyse, Medison, Merck-Serono, Novartis, Sanofi 
Genzyme, and Roche. FB is a board member for Neurology, Brain, Radiology, and MSJ; section editor for Neuroradiology; personal fees from Bayer, Biogen, Roche, IXICO, and GeNeuro; grants from Novartis, Teva Pharmaceuticals, Merck, Biogen, Innovative Medicines Initiative, General Electric Healthcare, UK MS Society, Dutch Foundation MS Research, NWO, and NIHR. JMS has received research funding and PET tracer from AVID Radiopharmaceuticals (a wholly owned subsidiary of Eli Lilly); has consulted for Roche, Eli Lilly, Biogen, Merck, and GE; received royalties from Oxford University Press and Henry Stewart Talks; given education lectures sponsored by Eli Lilly, Biogen, and GE; and served on a Data Safety Monitoring Committee for Axon Neuroscience SE. He is Chief Medical Officer for Alzheimer's Research UK.

Statistics and biometry One of the authors has significant statistical expertise.

Informed consent Written informed consent was obtained from all subjects and patients in this study.

Ethical approval Subject data in the 'reference dataset' were acquired under the ethical agreements in place for ADNI and TRACK-HD studies. All subjects in the 'patient dataset' gave their informed consent for all parts of the study under research ethical approval by the Queen Square ethics committee: References: 13 LO 0005 and 12 LO 1504.

Study subjects or cohorts overlap Some study subjects or cohorts have been previously reported in:

Tabrizi SJ, Reilmann R, Roos RA, et al (2012) Potential endpoints for clinical trials in premanifest and early Huntington's disease in the TRACK-HD study: analysis of 24 month observational data. Lancet Neurol 11:42-53.

Studies related to the Alzheimer's Disease Neuroimaging Initiative

\author{
Methodology \\ - retrospective \\ - cross-sectional study/diagnostic \\ - multicentre study
}

Open Access This article is licensed under a Creative Commons Attribution 4.0 International License, which permits use, sharing, adaptation, distribution and reproduction in any medium or format, as long as you give appropriate credit to the original author(s) and the source, provide a link to the Creative Commons licence, and indicate if changes were made. The images or other third party material in this article are included in the article's Creative Commons licence, unless indicated otherwise in a credit line to the material. If material is not included in the article's Creative Commons licence and your intended use is not permitted by statutory regulation or exceeds the permitted use, you will need to obtain permission directly from the copyright holder. To view a copy of this licence, visit http://creativecommons.org/licenses/by/4.0/.

\section{References}

1. Dubois B, Feldman HH, Jacova C et al (2007) Research criteria for the diagnosis of Alzheimer's disease: revising the NINCDSADRDA criteria. Lancet Neurol 6:734-746. https://doi.org/10. 1016/S1474-4422(07)70178-3
2. Jack CR, Bennett DA, Blennow K et al (2018) NIA-AA research framework: toward a biological definition of Alzheimer's disease. Alzheimers Dement 14:535-562

3. Duchesne S, Caroli A, Geroldi C, Barillot C, Frisoni GB, Collins DL (2008) MRI-based automated computer classification of probable AD versus normal controls. IEEE Trans Med Imaging 27:509520. https://doi.org/10.1109/TMI.2007.908685

4. ten Kate M, Barkhof F, Boccardi M et al (2017) Clinical validity of medial temporal atrophy as a biomarker for Alzheimer's disease in the context of a structured 5-phase development framework. Neurobiol Aging. https://doi.org/10.1016/j.neurobiolaging.2016. 05.024

5. Winblad B, Amouyel P, Andrieu S et al (2016) Defeating Alzheimer's disease and other dementias: a priority for European science and society. Lancet Neurol 15:455-532

6. Scheltens P, Leys D, Barkhof F et al (1992) Atrophy of medial temporal lobes on MRI in Alzheimer's disease and normal ageing: diagnostic value and neuropsychological correlates. J Neurol Neurosurg Psychiatry 55:967-972

7. Boutet $\mathrm{C}$, Chupin $\mathrm{M}$, Colliot $\mathrm{O}$ et al (2012) Is radiological evaluation as good as computer-based volumetry to assess hippocampal atrophy in Alzheimer's disease? Neuroradiology 54:1321-1330. https://doi.org/10.1007/s00234-012-1058-0

8. Vernooij MW, Pizzini FB, Schmidt R et al (2019) Dementia imaging in clinical practice: a European-wide survey of 193 centres and conclusions by the ESNR working group. Neuroradiology. https:// doi.org/10.1007/s00234-019-02188-y

9. Pasquier F, Leys D, Weerts JGE, Mounier-Vehier F, Barkhof F, Scheltens P (1996) Inter-and intraobserver reproducibility of cerebral atrophy assessment on MRI scans with hemispheric infarcts. Eur Neurol. https://doi.org/10.1159/000117270

10. Koedam ELGE, Lehmann M, van der Flier WM et al (2011) Visual assessment of posterior atrophy development of a MRI rating scale. Eur Radiol 21:2618-2625. https://doi.org/10.1007/s00330-0112205-4

11. Salvatore C, Cerasa A, Castiglioni I (2018) MRI characterizes the progressive course of $\mathrm{AD}$ and predicts conversion to Alzheimer's dementia 24 months before probable diagnosis. Front Aging Neurosci 10. https://doi.org/10.3389/fnagi.2018.00135

12. Harper L, Barkhof F, Fox NC, Schott JM (2015) Using visual rating to diagnose dementia: a critical evaluation of MRI atrophy scales. J Neurol Neurosurg Psychiatry 86:1225-1233

13. Harper L, Fumagalli GG, Barkhof F et al (2016) MRI visual rating scales in the diagnosis of dementia: evaluation in 184 post-mortem confirmed cases. Brain 139:1211-1225. https://oi.org/10.1093/ brain/aww005

14. Fox NC, Black RS, Gilman S et al (2005) Effects of A $\beta$ immunization (AN1792) on MRI measures of cerebral volume in Alzheimer disease. Neurology 64:1563-1572. https://doi.org/10. 1212/01.WNL.0000159743.08996.99

15. Schwarz AJ, Sundell KL, Charil A et al (2019) Magnetic resonance imaging measures of brain atrophy from the EXPEDITION3 trial in mild Alzheimer's disease. Alzheimer's Dement (N Y) 5:328-337. https://doi.org/10.1016/j.trci.2019.05.007

16. Brewer JB (2009) Fully-automated volumetric MRI with normative ranges: translation to clinical practice. Behav Neurol 21:21-28. $\mathrm{https}$ ://doi.org/10.3233/BEN-2009-0226

17. Hedderich DM, Spiro JE, Goldhardt $O$ et al (2018) Increasing diagnostic accuracy of mild cognitive impairment due to Alzheimer's disease by user-independent, web-based whole-brain Volumetry. J Alzheimers Dis 65:1459-1467. https://doi.org/10.3233/JAD180532

18. Ross DE, Ochs AL, Seabaugh JM, Shrader CR; Alzheimer's Disease Neuroimaging Initiative (2013) Man versus machine: comparison of radiologists' interpretations and NeuroQuant ${ }^{\circledR}$ volumetric analyses of brain MRIs in patients with traumatic brain injury. $\mathrm{J}$ 
Neuropsychiatry Clin Neurosci 25:32-39. https://doi.org/10.1176/ appi.neuropsych.11120377

19. Vernooij MW, Jasperse B, Steketee R et al (2018) Automatic normative quantification of brain tissue volume to support the diagnosis of dementia: a clinical evaluation of diagnostic accuracy. Neuroimage Clin 20:374-379. https://doi.org/10.1016/J.NICL. 2018.08 .004

20. Heckemann RA, Hammers A, Rueckert D, Aviv RI, Harvey CJ, Hajnal JV (2008) Automatic volumetry on MR brain images can support diagnostic decision making. BMC Med Imaging 8:9. https://doi.org/10.1186/1471-2342-8-9

21. Hedderich DM, Dieckmeyer M, Andrisan T et al (2020) Normative brain volume reports may improve differential diagnosis of dementing neurodegenerative diseases in clinical practice. Eur Radiol 30:2821-2829. https://doi.org/10.1007/s00330-019-066020

22. Koga H, Yuzuriha T, Yao H et al (2002) Quantitative MRI findings and cognitive impairment among community dwelling elderly subjects. J Neurol Neurosurg Psychiatry 72:737-741. https://doi.org/ 10.1136/JNNP.72.6.737

23. McEvoy LK, Brewer JB (2010) Quantitative structural MRI for early detection of Alzheimer's disease. Expert Rev Neurother 10: 1675. https://doi.org/10.1586/ERN.10.162

24. Goodkin O, Pemberton H, Vos SB et al (2019) The quantitative neuroradiology initiative framework: application to dementia. Br J Radiol. https://doi.org/10.1259/bjr.20190365

25. Cardoso MJ, Modat M, Wolz R, et al (2015) Geodesic Information Flows: Spatially-Variant Graphs and Their Application to Segmentation and Fusion. IEEE Trans Med Imaging 34:19761988. https://doi.org/10.1109/TMI.2015.2418298

26. Sudre CH, Gomez Anson B, Davagnanam I et al (2018) Bullseye's representation of cerebral white matter hyperintensities. J Neuroradiol 45:114-122. https://doi.org/10.1016/j.neurad.2017. 10.001

27. Tabrizi SJ, Reilmann R, Roos RA et al (2012) Potential endpoints for clinical trials in premanifest and early Huntington's disease in the TRACK-HD study: analysis of 24 month observational data. Lancet Neurol 11:42-53. https://doi.org/10.1016/S1474-4422(11) 70263-0

28. Meeter LH, Dopper EG, Jiskoot LC et al (2016) Neurofilament light chain: a biomarker for genetic frontotemporal dementia. Ann Clin Transl Neurol 3:623-636. https://doi.org/10.1002/acn3.325

29. Bocchetta M, Cardoso MJ, Cash DM, Ourselin S, Warren JD, Rohrer JD (2016) Patterns of regional cerebellar atrophy in genetic frontotemporal dementia. Neuroimage Clin 11:287-290. https:// doi.org/10.1016/j.nicl.2016.02.008

30. Pardini M, Sudre CH, Prados F et al (2016) Relationship of grey and white matter abnormalities with distance from the surface of the brain in multiple sclerosis. J Neurol Neurosurg Psychiatry 87:12121217. https://doi.org/10.1136/jnnp-2016-313979

31. Jorge Cardoso M, Leung K, Modat M et al (2013) Performancebased multi-classifier decision fusion for atlas-based segmentation of biomedical images Image Guidance Laboratories, Department of Neurosurgery, Stanford University, Stanford, CA Department of Computer Science, Stanford University, Stan. J Med Radiat Sci 25:404-407. https://doi.org/10.1109/TPAMI.2003.1159950

32. Ahmadi-Abhari S, Guzman-Castillo M, Bandosz P et al (2017) Temporal trend in dementia incidence since 2002 and projections for prevalence in England and Wales to 2040: modelling study. BMJ 358:j2856. https://doi.org/10.1136/bmj.j2856

33. Brewer JB, Magda S, Airriess C, Smith ME (2009) Fullyautomated quantification of regional brain volumes for improved detection of focal atrophy in Alzheimer disease. AJNR Am J Neuroradiol 30:578-580. https://doi.org/10.3174/ajnr.A1402

34. Struyfs H, Sima DM, Wittens M et al (2020) Automated MRI volumetry as a diagnostic tool for Alzheimer's disease: validation of icobrain dm. Neuroimage Clin 26:102243. https://doi.org/10. 1016/j.nicl.2020.102243

Publisher's note Springer Nature remains neutral with regard to jurisdictional claims in published maps and institutional affiliations. 


\section{Affiliations}

Hugh G. Pemberton ${ }^{1,2,3}$ (D) Olivia Goodkin ${ }^{1,2} \cdot$ Ferran Prados $^{1,4} \cdot$ Ravi K. Das $^{5} \cdot$ Sjoerd B. Vos ${ }^{1,2} \cdot$ James Moggridge ${ }^{2,6} \cdot$ William Coath ${ }^{3} \cdot$ Elizabeth Gordon $^{3} \cdot$ Ryan Barrett $^{7} \cdot$ Anne Schmitt $^{2,6} \cdot$ Hefina Whiteley-Jones $^{7} \cdot$ Christian Burd $^{8}$. Mike P. Wattjes ${ }^{9} \cdot$ Sven Haller $^{10} \cdot$ Meike W. Vernooij $^{11,12} \cdot$ Lorna Harper $^{3} \cdot$ Nick C. Fox $^{3} \cdot$ Ross W. Paterson ${ }^{3}$. Jonathan M. Schott ${ }^{3} \cdot$ Sotirios Bisdas ${ }^{2,6} \cdot$ Mark White $^{13} \cdot$ Sebastien Ourselin $^{14} \cdot$ John S. Thornton $^{2,6}$. Tarek A. Yousry ${ }^{2,6} \cdot$ M. Jorge Cardoso ${ }^{14}$. Frederik Barkhof ${ }^{1,2,6,15} \cdot$ for the Alzheimer's Disease Neuroimaging Initiative

1 Centre for Medical Image Computing (CMIC), Department of Medical Physics and Bioengineering, University College London, London, UK

2 Neuroradiological Academic Unit, UCL Queen Square Institute of Neurology, University College London, London, UK

3 Dementia Research Centre, UCL Queen Square Institute of Neurology, University College London, London, UK

4 Universitat Oberta de Catalunya, Barcelona, Spain

5 Clinical, Educational and Health Psychology, University College London, London, UK

6 Lysholm Department of Neuroradiology, National Hospital for Neurology and Neurosurgery, UCLH NHS Foundation Trust, London, UK

7 Department of Neuroradiology, Brighton and Sussex University Hospitals, Brighton, UK
8 Guy's and St Thomas' NHS Foundation Trust, London, UK

9 Department of Diagnostic and Interventional Neuroradiology, Hannover Medical School, Hannover, Germany

10 Department of Surgical Sciences, Radiology, Uppsala University, Uppsala, Sweden

11 Department of Radiology and Nuclear Medicine, Erasmus MC University Medical Center, Rotterdam, the Netherlands

12 Department of Epidemiology, Erasmus MC University Medical Center, Rotterdam, the Netherlands

13 Digital Services, University College London Hospital, London, UK

14 School of Biomedical Engineering and Imaging Sciences, King's College London, London, UK

15 Radiology \& Nuclear Medicine, VU University Medical Center, Amsterdam, the Netherlands 